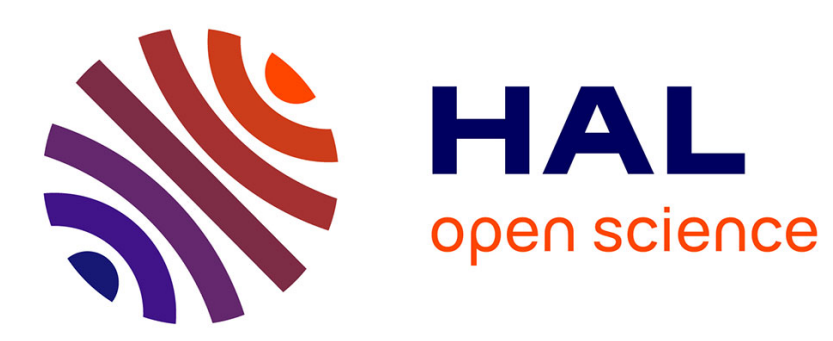

\title{
Mechanistic Aspects of the Photophysics of UVA Filters Based on Meldrum Derivatives
}

\author{
Josene M Toldo, Mariana T Do Casal, Mario Barbatti
}

\section{To cite this version:}

Josene M Toldo, Mariana T Do Casal, Mario Barbatti. Mechanistic Aspects of the Photophysics of UVA Filters Based on Meldrum Derivatives. Journal of Physical Chemistry A, 2021, 125 (25), pp.5499-5508. 10.1021/acs.jpca.1c03315 . hal-03276126

\section{HAL Id: hal-03276126 \\ https://hal.science/hal-03276126}

Submitted on 1 Jul 2021

HAL is a multi-disciplinary open access archive for the deposit and dissemination of scientific research documents, whether they are published or not. The documents may come from teaching and research institutions in France or abroad, or from public or private research centers.
L'archive ouverte pluridisciplinaire HAL, est destinée au dépôt et à la diffusion de documents scientifiques de niveau recherche, publiés ou non, émanant des établissements d'enseignement et de recherche français ou étrangers, des laboratoires publics ou privés. 


\title{
Mechanistic Aspects of the Photophysics of UVA Filters Based on Meldrum Derivatives
}

\author{
Josene M. Toldo,* Mariana T. do Casal, and Mario Barbatti*
}

Cite This: J. Phys. Chem. A 2021, 125, 5499-5508

Read Online

ABSTRACT: Skin photoprotection against UVA radiation is crucial, but it is hindered by the sparsity of approved commercial UVA filters. Sinapoyl malate (SM) derivatives are promising candidates for a new class of UVA filters. They have been previously identified as an efficient photoprotective sunscreen in plants due to their fast nonradiative energy dissipation. Combining experimental and computational results, in our previous letter (J. Phys. Chem. Lett. 2021, 12, 337-344) we showed that coumaryl Meldrum (CMe) and sinapoyl Meldrum (SMe) are outstanding candidates for UVA filters in sunscreen formulations. Here, we deliver a comprehensive computational characterization of the excitedstate dynamics of these molecules. Using reaction pathways and excited-state dynamics simulations, we could elucidate the photodeactivation mechanism of

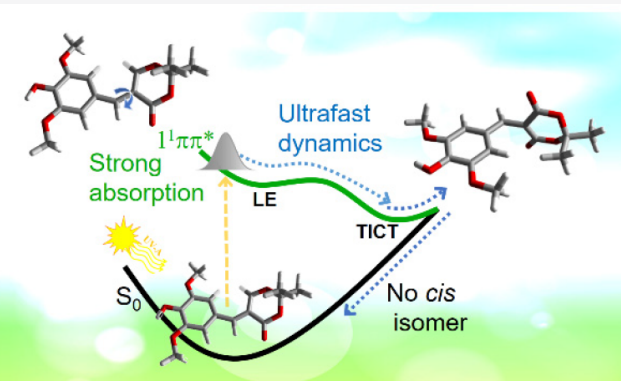
these molecules. Upon photoexcitation, they follow a two-step logistic decay. First, an ultrafast and efficient relaxation stabilizes the excited state alongside a $90^{\circ}$ twisting around the allylic double bond, giving rise to a minimum with a twisted intramolecular excitedstate (TICT) character. From this minimum, internal conversion to the ground state occurs after overcoming a $0.2 \mathrm{eV}$ barrier. Minor differences in the nonradiative decay and fluorescence of CMe and SMe are associated with an additional minimum present only in the latter.

\section{INTRODUCTION}

Despite the essential role of solar radiation in sustain life on Earth, it is well known that overexposure to ultraviolet (UV) radiation can photodamage the skin and cause DNA mutation. ${ }^{1,2}$ While a negligible amount of UVC radiation reaches the Earth, the amount of UVA (400-315 nm) and UVB $(315-280 \mathrm{~nm})$ radiation is substantial. ${ }^{3,4}$ Although UVA radiation does not directly damage the DNA, the amount of UVA radiation reaching the Earth is 10 times larger than that of UVB. Moreover, UVA penetrates deeper into the skin, inducing profound alterations of the dermal connective tissue. ${ }^{5-7}$ Most commercially available sunscreens combine both UVA and UVB chemical filters to provide broad-spectrum protection. The big issue is that popular UVA absorbers, like avobenzone, are not photostable. $^{8,9}$ Indeed, a good candidate for an efficient UV chemical filter should have an efficient nonradiative decay and long-term photostability to dissipate the excess of absorbed energy safely (without forming photoproducts) and quickly regenerate the original ground state. ${ }^{3}$ Yet, it should have a strong UV absorption with a large absorption cross-section. ${ }^{3}$

The inspiration to develop a new generation of UV filters complying with these features comes from nature. Plants also need a defense mechanism against UV radiation because the excess of UVB radiation can cause them several damaging effects. ${ }^{3,10}$ Sinapoyl malate (SM), a natural absorbing compound, was found to be involved in the mechanism protecting Arabidopsis thaliana leaves against UVB damage. ${ }^{11-13}$ Inspired by SM, other sinapate esters (methyl and ethyl sinapate) were identified as promising candidates for natural sunscreens by Stavros and collaborators ${ }^{14-16}$ and Zhao and collaborators. ${ }^{17}$ In these molecules, the ultrafast decay is mediated by a conical intersection between the first excited state $\left(S_{1}\right)$ and the ground state $\left(S_{0}\right)$ through a trans/cis isomerization across the allylic bond. ${ }^{11,12,18}$ As the trans isomer is regenerated in the $S_{0}$ state, the cis isomer is also formed, which has been reported to have high genotoxicity in related cinnamate filters. ${ }^{19,20}$ Therefore, avoiding the cis isomer's formation became a drive to design new nature-inspired sunscreen molecules.

A way to overcome the cis isomer's formation is to design a cinnamate or sinapate derivative containing symmetric substitution around the allylic double bond. The first attempt toward this goal was reported by Horbury et al. ${ }^{21}$ Using transient absorption spectra (TAS) and steady-state spectroscopy, they investigated the ultrafast decay of a cis/trans invariant sinapate ester, which has shown promising results on the impact of symmetric substitution around the allylic double bond. This work has inspired the design of other cis/trans invariant derivatives such as coumaryl Meldrum (CMe) and sinapoyl

Received: April 13, 2021

Revised: May 27, 2021

Published: June 20, 2021 
Meldrum (SMe) (see Figure 1). The Meldrum series presents all of the characteristics needed to fulfill the requests for a new a)

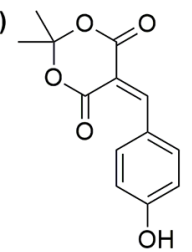

b)

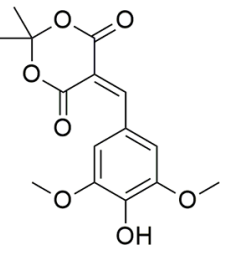

Figure 1. Molecular structure of (a) coumaryl Meldrum (CMe) and (b) sinapoyl Meldrum (SMe) investigated in this work.

generation of UVA filters: a broad and robust absorption spectrum in the UVA region, an ultrafast decay that regenerates the initial ground state, high photostability, and no endocrine disruption activity.

In a previous letter, ${ }^{22}$ we combined experimental and theoretical efforts to characterize the $\mathrm{CMe}$ and $\mathrm{SMe}$ photophysics and photochemistry. In the present work, we use quantum-chemical calculations to perform a comprehensive analysis of these systems' photodynamics. We characterize the reaction pathways, show how they induce a logistic decay of the excited-state population, and discuss diverse effects impacting the time constants.

\section{COMPUTATIONAL DETAILS}

Static Calculations. The ground and first excited states of $\mathrm{CMe}$ and SMe were optimized using density functional theory (DFT) and time-dependent DFT (TD-DFT) with the $\omega \mathrm{B} 97 \mathrm{XD}$ functional. ${ }^{23}$ For comparison, the B3LYP ${ }^{24,25}$ functional using D3 dispersion corrections ${ }^{26}$ was also used. All optimizations were done using the $c c-p V D Z{ }^{27}$ basis set and linear response Polarizable Continuum Model (LR-PCM) ${ }^{28}$ with ethanol as implicit solvent. To evaluate the effect of the dielectric constant of the solvent, pentyl ethanoate (which has similar properties as caprylic capric triglyceride (CCT), experimentally employed) was also used. Vertical and adiabatic energies were computed using the aug-cc-pVTZ basis set. All (TD)-DFT calculations were done using Gaussian 16 rev a03. ${ }^{29}$

The influence of intermolecular hydrogen bonds between $\mathrm{CMe}$ and SMe and ethanol was evaluated using microsolvation. We included three and four ethanol molecules during the optimization to obtain the vertical transition energies for $\mathrm{CMe}$ and $\mathrm{SMe}$, respectively. The same calculation level was utilized ( $\omega$ B97XD/aug-cc-pVTZ// $\omega$ B97XD/cc-pVDZ and PCM/ ethanol model).

The geometries of the minimal energy crossing points (MECP) between $S_{1}$ and $S_{0}$ were located using the penalty function method implemented in the Conical Intersection Optimizer (CIOpt) software by Levine and co-workers ${ }^{30,31}$ and adapted by us to work with Gaussian software. Linear interpolations using natural internal coordinates ${ }^{32}$ (LIIC) and rigid scan of a few coordinates were used to estimate the energy barriers in the excited state, both at the $\omega \mathrm{B} 97 \mathrm{XD} / \mathrm{cc}-\mathrm{pVDZ}$ level using PCM/ethanol.

The structures and energies obtained for the $S_{1} / S_{0}$ MECP were evaluated by comparison with multiconfigurational calculations. This allows us to verify the multiconfigurational character of the electronic states. For $\mathrm{CMe}$, the $S_{1}$ minimum and $S_{1} / S_{0}$ conical intersection geometries were optimized using a state-averaged complete active space self-consistent field (SACASSCF). ${ }^{33}$ The corresponding vertical energies were computed using multistate complete active space second-order perturbation (MS-CASPT2). ${ }^{34}$ The ANO-L-VDZP ${ }^{35}$ basis set was used. For the optimizations, we selected an active space composed of 6 electrons in 6 orbitals and 4 states in the state average procedure. This space included the $\pi$ and $\pi^{*}$ orbitals with the highest fractional occupations. To select this active space, we first did CASPT2//CASSCF single-point calculations on $\omega \mathrm{B} 97 \mathrm{XD} / \mathrm{cc}-\mathrm{pVDZ}$-optimized geometries for $\mathrm{S}_{0}, \mathrm{~S}_{1}$, and $\mathrm{S}_{1} /$ $\mathrm{S}_{0}$ MECP of CMe using a larger active space. This active space was composed of 14 electrons in 12 orbitals and included the 2 carbonyl lone pairs and all $\pi$ and $\pi^{*}$ orbitals (except the highest occupied $\pi$ and lowest occupied $\pi^{*}$ orbitals). These calculations were averaged over five states. The default IPEA ${ }^{36}$ shift $(0.25 \mathrm{au})$ and imaginary shift ${ }^{37}$ of 0.1 au were used. For SMe, the vertical excitations from the $S_{0}$ state were obtained using a larger active space (16 electrons in 13 orbitals). All CASPT2//CASSCF calculations were done in the gas phase using OpenMolcas v.19.11 (tag 283-ge7efbbb). ${ }^{38}$

The charge-transfer character of the excited states was evaluated by computing the charge-transfer number $q(\mathrm{CT})$ as implemented in the TheoDORE program. ${ }^{39}$ Charge-transfer numbers are calculated as partial summations over squared transition density matrix elements of molecular fragments. As a result, a value of $q(\mathrm{CT})$ near one represents a complete charge separation, whereas $q(\mathrm{CT})$ near zero represents a locally excited (LE) state.

Excited-State Dynamics and Spectrum Simulations. The initial conditions for the dynamics were obtained by first simulating the absorption spectra of CMe and SMe using TD$\omega \mathrm{B} 97 \mathrm{XD} / \mathrm{cc}-\mathrm{pVDZ}$ and implicit PCM/ethanol. The initial conditions were generated using the harmonic oscillator Wigner distribution based on the $S_{0}$ normal modes. A set of 500 and 300 initial conditions was generated for $\mathrm{CMe}$ and $\mathrm{SMe}$, respectively. Vertical excitation energies and oscillator strengths for the first 10 excited singlet states were computed for $\mathrm{CMe}$, while 6 excited states were computed for SMe. The photoabsorption spectra of $\mathrm{CMe}$ and $\mathrm{SMe}$ were simulated using the nuclear ensemble approach. ${ }^{40}$ Spectrum simulation and excited-state dynamics were done using NEWTON-X v.2.2-B09 ${ }^{41}$ interfaced with Gaussian $09 .^{42}$

Excited-state dynamics simulations were performed using TD- $\omega$ B97XD/cc-pVDZ in implicit ethanol. For CMe, adiabatic dynamic on the $S_{1}$ surface was done considering a total of 70 trajectories. The initial conditions were selected from the simulated spectrum within the $3.8 \pm 0.3 \mathrm{eV}$ spectral window. The velocity Verlet ${ }^{43}$ algorithm with a time step of 0.5 fs was used for the integration of Newton's equations. For SMe, due to the energetic proximity between the $S_{2}$ and the $S_{1}$ states, a nonadiabatic dynamics simulation was done using the decoherence-corrected $^{44}$ fewest switches surface hopping (DC-FSSH) approach. ${ }^{45} \mathrm{~A}$ total of 67 trajectories starting from the bright $S_{1}$ state was considered. The initial conditions for the dynamics were sampled within the $3.5 \pm 0.3 \mathrm{eV}$ spectral window. For both molecules, we considered that a trajectory went back to the ground state when the SCF did not converge anymore. At this point, the molecules reach a region with a high multiconfigurational character and have a small energy gap $(<0.3$ $\mathrm{eV}$ ) with the $S_{0}$ state.

\section{RESULTS AND DISCUSSION}

States Characterization by Static Calculations. The optimized geometries of CMe and SMe in the $S_{0}$ and $S_{1}$ states are shown in Figure 2. In the ground state, both molecules are 

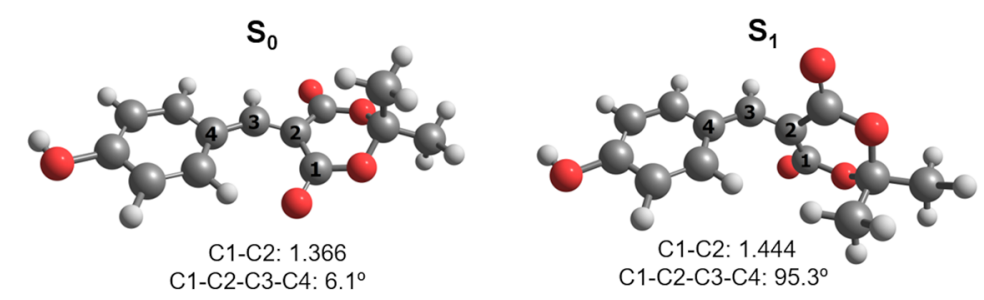

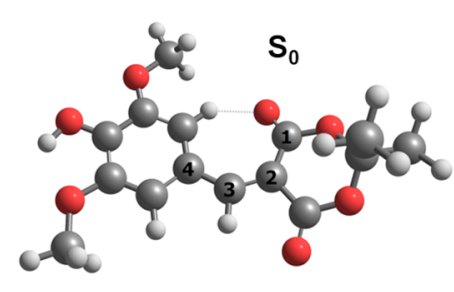

C1-C2: 1.366 C1-C2-C3-C4: $5.5^{\circ}$

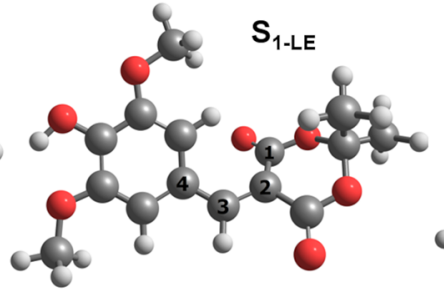

C1-C2: 1.429

C1-C2-C3-C4: $39.2^{\circ}$

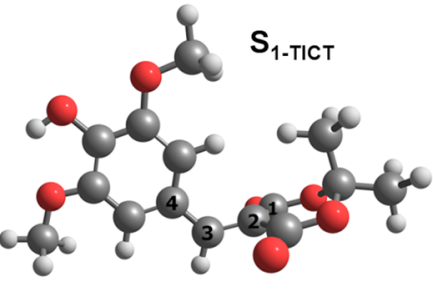

C1-C2: 1.444 C1-C2-C3-C4: $94.9^{\circ}$

Figure 2. Optimized geometries for CMe (above) and SMe (below) in the $S_{0}$ and $S_{1}$ states calculated at $\omega \mathrm{B} 97 \mathrm{XD} / \mathrm{cc}-\mathrm{pVDZ}$ using PCM/ethanol. Inserts show the main structural changes ( $\mathrm{C} 2-\mathrm{C} 3$ bond lengths in Ångströms, and $\mathrm{C} 1-\mathrm{C} 2-\mathrm{C} 3-\mathrm{C} 4$ dihedral angle in degrees) going from the ground to the first excited state.
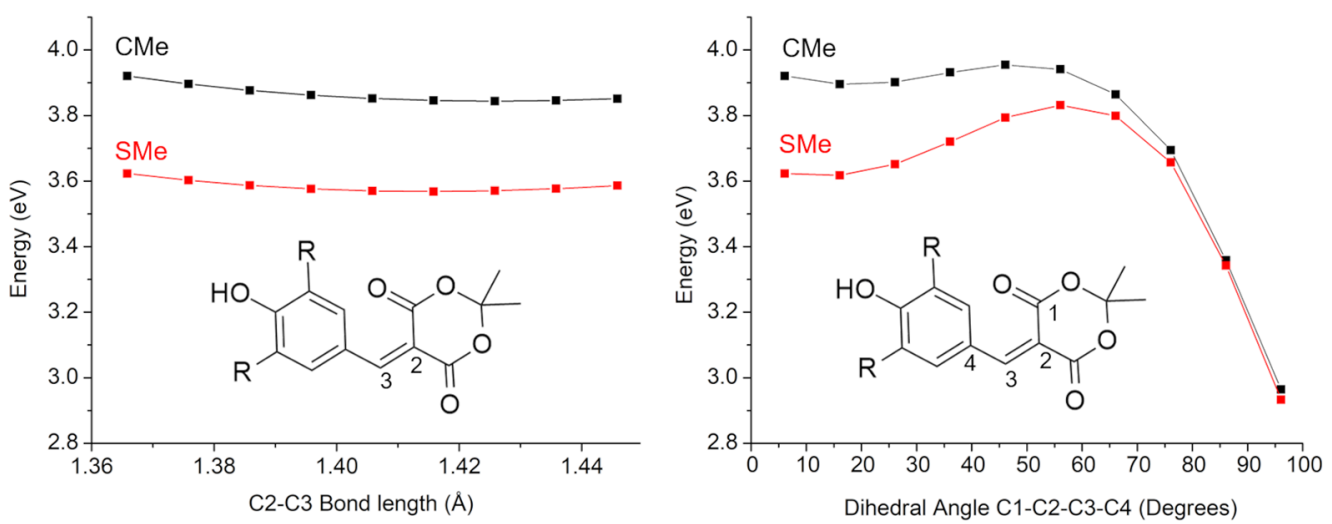

Figure 3. Potential energy curves for the rigid scans of the $\mathrm{C} 2-\mathrm{C} 3$ stretching (left) and $\mathrm{C} 1-\mathrm{C} 2-\mathrm{C} 3-\mathrm{C} 4$ torsion (right), starting from the vertically excited $\mathrm{S}_{1}$ to the $\mathrm{C} 2-\mathrm{C} 3$ and dihedral angle $\varphi$ corresponding to those at the $\mathrm{S}_{1 \text {-TICT }}$ minimum. Geometries and energies computed at the $\omega \mathrm{B} 97 \mathrm{XD} / \mathrm{cc}-$ $\mathrm{pVDZ}$ level in PCM/ethanol. For CMe and $\mathrm{SMe}, \mathrm{R}=\mathrm{H}$ and $\mathrm{OCH}_{3}$, respectively.

Table 1. Vertical Excitations $\left(\Delta E_{\text {vert }}\right)$, Adiabatic Energies $\left(\Delta E_{\text {adiab }}\right)$, Emission Energies $\left(\Delta E_{\text {em }}\right)$, and Oscillator Strengths (in parentheses) Calculated for the Lowest Singlet Excited States of CMe and SMe at TD-DFT ${ }^{a}$ and MS-CASPT2 ${ }^{b}$

\begin{tabular}{|c|c|c|c|c|c|c|c|c|c|}
\hline \multirow{3}{*}{$\mathrm{CMe}$} & \multirow{4}{*}{$\mathrm{S}_{1}$} & \multicolumn{6}{|c|}{ TD- $\omega$ B97XD } & \multirow{2}{*}{\multicolumn{2}{|c|}{$\frac{\text { MS-CASPT2 }}{\Delta E_{\text {vert }}(\mathrm{eV})\left(\mathrm{S}_{0} \text { geom }\right)}$}} \\
\hline & & \multicolumn{2}{|c|}{$\Delta E_{\text {vert }}(\mathrm{eV})\left(\mathrm{S}_{0}\right.$ geom $)$} & \multicolumn{2}{|c|}{$\Delta E_{\mathrm{ad}}(\mathrm{eV})^{f}\left(\mathrm{~S}_{\mathrm{n}}\right.$ geom $)$} & \multicolumn{2}{|c|}{$\Delta E_{\mathrm{em}}(\mathrm{eV})\left(\mathrm{S}_{1}\right.$ geom $)$} & & \\
\hline & & $\pi \pi^{*}$ & $3.83(0.876)$ & $\pi \pi^{*}$ & $2.51(0.000)$ & $\pi \pi^{*}$ & $0.79(0.000)$ & $\pi \pi^{*}$ & $3.68(0.735)$ \\
\hline & & & $3.31^{c}$ & & & & & & \\
\hline & $\mathrm{S}_{2}$ & $\pi \pi^{*}$ & $4.62(0.007)$ & $\pi \pi^{*}$ & $4.25(0.051)$ & $\mathrm{n} \pi^{*}$ & $3.14(0.032)$ & $\mathrm{n} \pi^{*}$ & $4.59(0.002)$ \\
\hline & $\mathrm{S}_{3}$ & $\mathrm{n} \pi^{*}$ & $4.70(0.004)$ & $\mathrm{n} \pi^{*}$ & $4.30(0.016)$ & $\pi \pi^{*}$ & $3.64(0.024)$ & $\pi \pi^{*}$ & $5.12(0.005)$ \\
\hline \multirow[t]{5}{*}{$\mathrm{SMe}$} & $S_{1-\mathrm{LE}}$ & $\pi \pi^{*}$ & $3.57(0.666)$ & $\pi \pi^{*}$ & $3.18(0.528)$ & $\pi \pi^{*}$ & $2.77(0.528)$ & $\pi \pi^{*}$ & $3.41(0.668)$ \\
\hline & & & $3.05^{c}$ & & & & & & \\
\hline & $S_{\text {1-TICT }}$ & & & $\pi \pi^{*}$ & $2.49(0.000)$ & $\pi \pi^{*}$ & $0.82(0.000)$ & & \\
\hline & $\mathrm{S}_{2}$ & $\pi \pi^{*}$ & $3.86(0.018)$ & & $\mathrm{NO}^{d}$ & $\pi \pi^{*}$ & $2.76(0.017)^{e}$ & $\pi \pi^{*}$ & $4.04(0.080)$ \\
\hline & $\mathrm{S}_{3}$ & $\mathrm{n} \pi^{*}$ & $4.68(0.002)$ & $\mathrm{n} \pi^{*}$ & $4.29(0.000)$ & $\mathrm{n} \pi^{*}$ & $3.14(0.097)^{e}$ & $\mathrm{n} \pi^{*}$ & $4.57(0.001)$ \\
\hline
\end{tabular}

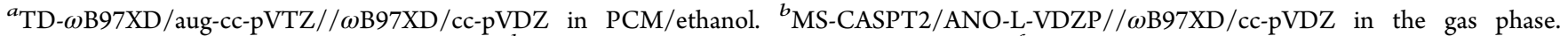
${ }^{c}$ Experimental values in ethanol from ref $22 .{ }^{d}$ Not obtained. ${ }^{e}$ Relative to $S_{1 \text {-TICT }}$ minimum. ${ }^{f}$ The adiabatic energies were calculated taking the difference between the energy of the optimized $S_{0}$ and the $S_{n}$ state (where $n=1,2$, or 3 ).

nearly planar. After excitation to the $S_{1}$ state, they undergo a remarkable geometrical change marked mainly by the stretching of the allylic $\mathrm{C} 2=\mathrm{C} 3$ bond and by the twist of the $\mathrm{C} 1-\mathrm{C} 2-$ C3-C4 dihedral angle $(\varphi)$ to around $90^{\circ}$. This global minimum has a strong charge-transfer character (as will be discussed later), and here it is named $S_{1-\text { TICT }}$. One can see that the methoxy groups present in SMe do not impact the geometries of $S_{0}$ and $S_{1 \text {-TICT }}$ In addition, a second $S_{1}$ minimum was located for $S M e$. This minimum, here named $S_{1-L E}$, presents a partially twisted geometry with a dihedral angle $\varphi$ around $40^{\circ}$, higher energy, and smaller charge-transfer character than at $S_{1 \text {-ТICT. }}$. 
A significant energy stabilization accompanies the changes in the $S_{1}$ state geometries. Figure 3 shows the energy variation along the two most significant structural changes going from the vertically excited $S_{1}$ state to the $S_{1-\text { TICT }}$ energy minimum of CMe. In these potential energy curves (PECs), we scanned two coordinates keeping all others fixed to their initial values. This procedure allows us to evaluate the individual effect of changing these coordinates on the energy. The PEC for the rigid scan of the $\mathrm{C} 2-\mathrm{C} 3$ stretching shows that this change has a minor effect on the stabilization of $\mathrm{CMe}$ and $\mathrm{SMe}$ in the $\mathrm{S}_{1}$ state. On the other hand, the increase in the $\mathrm{C} 1-\mathrm{C} 2-\mathrm{C} 3-\mathrm{C} 4$ dihedral angle largely stabilizes both molecules. One can see that although $\mathrm{SMe}$ in the vertically excited $S_{1}$ state has smaller energy when compared to $\mathrm{CMe}$, for the twisted geometries with $\varphi \approx 90^{\circ}$, the effect of the -OMe groups is negligible. Yet, the torsional barrier is larger for $\mathrm{SMe}(0.21 \mathrm{eV})$ than for $\mathrm{CMe}(0.06 \mathrm{eV})$, with the highest energy computed at $\varphi \approx 55^{\circ}$. The highest torsional barrier for $\mathrm{SMe}$ seems to be related to the appearance of a local minimum, $S_{1-\mathrm{LE}}$, not observed in CMe. The potential energy surface for these two structural changes, including the $S_{0}$ state, is shown in Figure $S 1$.

The vertical excitation, adiabatic, and emission energies computed at different levels are given in Table 1. Although the first vertical excitation calculated with B3LYP-D3 matches well with the experimental values in ethanol (see Table S1), this functional is not appropriate to describe the $S_{1}$ potential energy surface (PES) due to the charge-transfer character of the $S_{1}$ state. The overstabilization of charge-transfer states by hybrid functionals and the use of range-separated functionals (such as $\omega \mathrm{B} 97 \mathrm{XD})$ to overcome this problem is well established in the literature. ${ }^{46}$ Yet, there is a good agreement in the description of the vertical excitations between the TD- $\omega \mathrm{B} 97 \mathrm{XD}$ and the MSCASPT2 levels. Thus, we have chosen $\omega \mathrm{B} 97 \mathrm{XD}$ to explore the excited-state potential energy surfaces.

Table 1 shows that after photon absorption, both molecules are excited to the bright $S_{1}$ state, which has ${ }^{1} \pi \pi^{*}$ character, and it corresponds to the bright $\mathrm{V}$ state reported for similar molecules. ${ }^{11,47}$ The second vertically excited state also has a ${ }^{1} \pi \pi^{*}$ character and a small oscillator strength, and it can be related to the $\mathrm{V}^{\prime}$ state. The dark ${ }^{1} \mathrm{n} \pi^{*}$ state lies considerably higher in energy at the Franck-Condon (FC) region $(0.9 \mathrm{eV}$ for $\mathrm{CMe}$ and $1.1 \mathrm{eV}$ for SMe). Natural transition orbitals (NTOs) associated with the three lowest vertical transitions can be seen in Figure S2, while optimized geometries obtained for $S_{1}, S_{2}$, and $\mathrm{S}_{3}$ with their respective NTOs characterization can be seen in Figures S3 and S4.

As mentioned, after excitation to the bright $S_{1}$ state, a large geometrical change is observed and followed by a considerable energy stabilization of this state while the ground state is destabilized. At the $S_{1 \text {-TICT }}$ minimum, the energy gap with the ground state is only $0.8 \mathrm{eV}$ for both molecules, and the oscillator strength becomes zero (Table 1). After optimization, the $S_{2}$ and $\mathrm{S}_{3}$ states are stabilized by $\sim 0.4 \mathrm{eV}$. Their adiabatic energies are at least $1.7 \mathrm{eV}$ higher than the optimized $S_{1}$ state. Therefore, this hinders the possibility of internal conversion to the $n \pi^{*}$ state during the photorelaxation, as reported for other natural sunscreens. ${ }^{48-50}$

The three lowest singlet excited states were also characterized in terms of the charge-transfer character (Table S2). The global minimum, $\mathrm{S}_{1 \text {-TICT }}$, has a strong charge-transfer character with a $q(\mathrm{CT})$ number $>0.8$, which indicates that almost one unit of charge is transferred from one side to another side of the allylic bond. For CMe and SMe molecules, the $S_{1}$ state at the FC region (i.e., at the $S_{0}$ geometry) is mostly a local excitation (LE), although it has some considerable amount of charge transfer ( $q(\mathrm{CT})$ number $\approx 0.4$ ). At the partially twisted $\mathrm{S}_{1-\mathrm{LE}}$ minimum, $\mathrm{SMe}$ has a similar $\mathrm{CT}$ number to the $\mathrm{FC}$ region, suggesting that it remains a state with a local excited (LE) character.

The presence of this local minimum in SMe could account for the weak fluorescence (quantum yield $<1 \%$ in ethanol) and stimulated emission experimentally observed for $\mathrm{SMe}$ at $\sim 475$ nm. ${ }^{22}$ This partially twisted minimum has an emission wavelength of $448 \mathrm{~nm}(2.77 \mathrm{eV})$ and oscillator strength of 0.528 (Table 1) calculated at the TD- $\omega$ B97XD level. Although a small fraction of excited molecules could be trapped in the TICT minimum, emission is not expected from there since it has no oscillator strength. A much weaker fluorescence for $\mathrm{CMe}$ is experimentally detected and also reflected in the relative intensity of the weak stimulated emission features. This signal could be explained by the presence of a very shallow minimum with a partially twisted geometry not detected by our calculations.

The experiments reveal that for both molecules the groundstate recovery is not complete. Photostability experiments using a solar simulation show a minor reduction of absorbance of $0.9 \%$ and $2.0 \%$ for $\mathrm{CMe}$ and SMe, respectively. This indicates that a tinny fraction of the excited molecules may be trapped in an excited state or even undergo intersystem crossing to a triplet manifold. However, this result also indicates that the vast majority of the excited-state population should be deactivated via internal conversion (IC) to the ground state, reforming the original pattern molecule. Therefore, how does the internal conversion to the ground state occur?

The optimized geometries obtained for the $S_{1} / S_{0}$ MECP with TD-DFT and CASSCF can be seen in Figure S5, along with the TD-DFT and MS-CASPT2 energy gaps in Table S3. Although numerical differences in the bond lengths are observed when comparing both methods, there is a similar trend in the geometries: the geometries found for the $S_{1} / S_{0}$ crossing are similar to those found for the $S_{1}$ state with a torsional angle $\varphi$ around $95^{\circ}$ but with a larger $\mathrm{C} 3-\mathrm{C} 4$ bond length. It is worth noticing that although the torsional angle around the allylic bond resembles the ethylene case, ${ }^{51,52}$ there is a leading structural difference in the $S_{1} / S_{0}$ intersection when compared to the conical intersection in ethylene: no pyramidalization of the $\mathrm{C} 2$ or $\mathrm{C} 3$ atom was found for either CMe or SMe. This should be related to the charge-transfer character of the $S_{1}$ state in these molecules. While in ethylene, the pyramidalization at the conical intersection is a result of the change in the $\mathrm{sp}^{2}$ character (and therefore, the polarity) of one of the carbon atoms, in CMe and SMe, the difference in the polarity around the allylic bond is intrinsic in their geometries, and it is a direct result of the charge-transfer character of the excited state. ${ }^{53}$

Yet, both methods predict an MECP lying above the $S_{1-\text { TICT }}$ minimum. The energy difference between the $S_{1 \text {-TICT }}$ and the MECP is $\sim 0.2 \mathrm{eV}$ at both the TD-DFT and the CASPT2// $\omega \mathrm{B} 97 \mathrm{XD}$ levels (see Figure $\mathrm{S} 6$ for the active space used on these calculations). This energy difference is slightly higher $(0.37 \mathrm{eV})$ when computed at the $\operatorname{CASSCF}(6,6)$ geometries. Therefore, the uphill MECP is consistent with the experimental observation of an incomplete recovery of the ground state.

Potential Energy Curves. The potential energy curves (PECs) showing the deactivation path for $\mathrm{CMe}$ and $\mathrm{SMe}$ are illustrated in Figure 4. The PECs were constructed as linear interpolations in internal coordinates (LIIC) connecting the vertically excited $S_{1}$ state to the closest $S_{1}$ minima, the $S_{1-\mathrm{LE}}$ to $S_{1 \text {-TICT }}$ minimum (for $\mathrm{SMe}$ ), and the $S_{1 \text {-ТICT }}$ to the $S_{1} / S_{0}$ state 

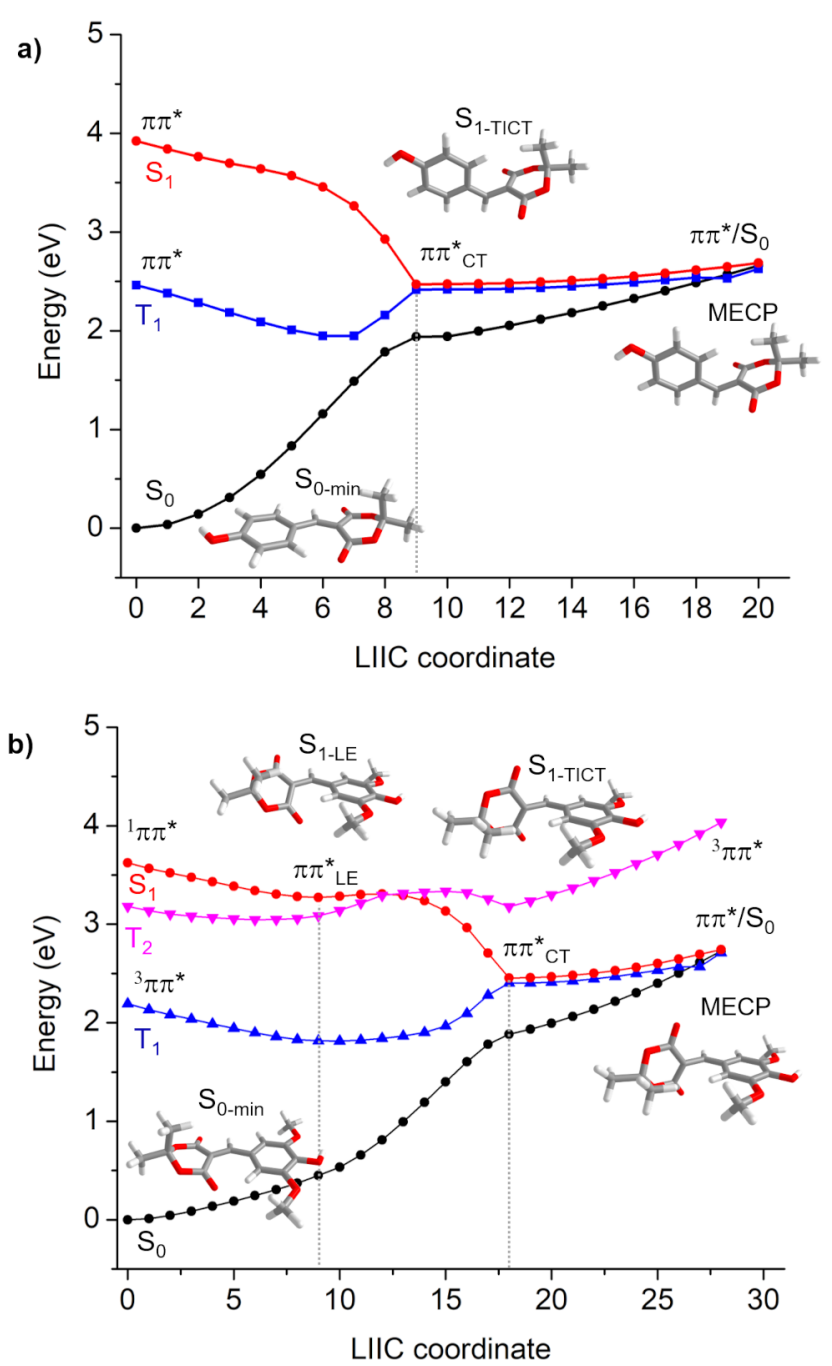

Figure 4. Potential energy curves calculated at TD- $\omega$ B97XD/cc-pVDZ using $\mathrm{PCM} /$ ethanol. (a) CMe: Linear interpolations in internal coordinates starting from $S_{1}$ vertically excited to the $S_{1 \text {-TICT }}$ minimum (shown until the dashed gray line) and from $S_{1-\text { TICT }}$ to $S_{1} / S_{0}$ MECP. (b) SMe: Linear interpolations in internal coordinates from $S_{1}$ vertically excited to $S_{1-\mathrm{LE}}$ minimum (shown until the first dashed gray line), from $S_{1-\mathrm{LE}}$ to $S_{1-\mathrm{TICT}}$ (until the second dashed gray line), and from $S_{1-\mathrm{TICT}}$ to $\mathrm{S}_{1} / \mathrm{S}_{0}$ MECP.

crossing. The closest triplet states calculated at the $S_{1}$ geometries are also shown. For CMe, one can see a large energy stabilization toward the formation of the $S_{1-\text { TICT }}$ minimum. After reaching this minimum, an energy plateau is observed. Closer inspection into the LIIC coordinates (presented in Figure S7) reveals an uphill $S_{1} / S_{0}$ MECP with an energy $0.2 \mathrm{eV}$ higher than the $S_{1-\text { TICT }}$ minimum. A crucial difference between $\mathrm{CMe}$ and $\mathrm{SMe}$ is that while a barrierless $S_{1}$ relaxation pathway was found for CMe, a flat region around the $S_{1-\mathrm{LE}}$ minimum is observed for SMe. A small energy barrier $(<2 \mathrm{kcal} / \mathrm{mol})$ was found connecting the $\mathrm{S}_{1 \text {-LE }}$ to the $\mathrm{S}_{1 \text {-TICT }}$ minimum. Similarly to $\mathrm{CMe}$, after reaching this global minimum, an uphill $S_{1} / S_{0}$ intersection was found 0.22 $\mathrm{eV}$ above the $\mathrm{S}_{1-\mathrm{TICT}}$ minimum.

Triplet States and Potential ISC. Aromatic carbonyl compounds are well known for having low-lying $\mathrm{n} \pi^{*}$ states, presenting intersystem crossing (ISC) as a competitive photophysical pathway. ${ }^{54}$ In fact, ISC was pointed out as a possible alternative relaxation pathway for the long-lived species in the experimental dynamics of CMe and SMe. ${ }^{22}$ However, this mechanism was not either confirmed or ruled out by the experiments since the weak fluorescence emission has precluded any reasonable fluorescence lifetime measures. We calculated the three lowest triplet states along with the PECs and evaluated them at the $S_{0}$ - and $S_{1}$-optimized geometries (Figure 4 and Table S4). For both molecules, the $\mathrm{T}_{1}, \mathrm{~T}_{2}$, and $\mathrm{T}_{3}$ states have $\pi \pi^{*}$ character. The first one, $T_{1}$, lies considerably below the $S_{1}$ state at the vertically excited geometry. For $\mathrm{SMe}, \mathrm{T}_{2}$ also lies below the vertically excited $S_{1}$ and the $S_{1-\mathrm{LE}}$ minimum. At the $S_{1-\text { TICT }}$ minimum, both molecules show the $T_{1}$ state near degenerate $(0.06 \mathrm{eV})$ with the $S_{1}\left(\pi \pi^{*}\right)$. Although close in energy, $S_{1}$ and $T_{1}$ have the same orbital symmetry, meaning that ISC is forbidden by El-Sayed's rule ${ }^{55}$ and, therefore, the spin-orbit coupling (SOC) should be minimal. Assuming a SOC of about $1.0 \mathrm{~cm}^{-1}$, a rough estimate of the ISC rate using the energy gap law ${ }^{56}$ (either in the strong or in the weak coupling regime) renders $k \simeq 10^{8}$ $\mathrm{s}^{-1}$, which is much slower than the estimated internal conversion rate. $^{22}$ Therefore, our calculations rule out the alternative relaxation pathway via ISC.

Solvent Effects. The solvent's influence on the absorption spectra and the $S_{1}$ minima of $\mathrm{CMe}$ and $\mathrm{SMe}$ was also investigated. Table 2 compares three different solvation schemes: PCM/pentyl ethanoate, PCM/ethanol, and microsolvated ethanol (PCM/ethanol plus 3 and 4 ethanol molecules for $\mathrm{CMe}$ and $\mathrm{SMe}$, respectively). The optimized geometries for the $S_{0}$ and $S_{1}$ states can be seen in Figure S8. Pentyl ethanoate was chosen because it presents similar dielectric constants and solvent properties as caprylic capric triglyceride (CCT) experimentally used. The main NTOs, which characterize the first lowest vertical excitations and the $S_{1}$ minima for CMe and $\mathrm{SMe}$, can be seen in Figures S9 and S10. One can see that the effect of solvent polarity is nearly negligible for both molecules. The inclusion of ethanol molecules has a more predominant effect: it slightly stabilizes the $\pi \pi^{*}$ states $\left(S_{1}\right.$ and $\left.S_{2}\right)$ and destabilizes the $n \pi^{*}$ state $\left(S_{3}\right)$ for both molecules. ${ }^{22}$ Such effect

Table 2. Vertical Excitations to the $S_{1}$ State $\left(\pi \pi^{*}\right)$ and $S_{1}$ Adiabatic Energies Calculated for CMe and SMe at $\omega$ B97XD/aug-ccpVTZ// 1 B97XD/cc-pVDZ Considering Different Solvation Schemes ${ }^{a}$

\begin{tabular}{clccccc} 
& \multicolumn{1}{c}{ State } & PCM PeEt & PCM Ethanol & PCM + EtOH & Exp. Ethanol & Exp. CCT \\
CMe & $S_{1 \text {-vert }\left(\pi \pi^{*}\right)}$ & $3.85(0.887)$ & $3.83(0.876)$ & $3.65(0.996)$ & 3.31 & 3.42 \\
& $S_{1}$-adiab TICT $\left(\pi \pi^{*}\right)$ & $2.44(0.000)$ & $2.51(0.000)$ & $2.58(0.000)$ & & 3.05 \\
SMe & $S_{0 \text {-vert }\left(\pi \pi^{*}\right)}$ & $3.57(0.794)$ & $3.57(0.666)$ & $3.43(0.917)$ & 3.13 \\
& $S_{1}$-adiab LE $\left(\pi \pi^{*}\right)$ & $3.21(0.457)$ & $3.18(0.528)$ & $3.00(0.649)$ & & \\
& $S_{1}$-adiab TICT $\left(\pi \pi^{*}\right)$ & $2.43(0.000)$ & $2.49(0.000)$ & $2.45(0.000)$ &
\end{tabular}

${ }^{a} \mathrm{PCM}$ (ethanol and pentyl ethanoate (PeEt) and microsolvation (PCM plus 3 and 4 ethanol molecules for CMe and SMe, respectively). Experimental values for the experimental absorption maxima in ethanol and CCT are also shown. ${ }^{22}{ }^{2}$ Pentyl ethanoate $(\varepsilon=4.7)$ instead of CCT $(\varepsilon$ = 3.93). 
was also reported by Liu et al. for a similar system (sinapic diacid, SA). ${ }^{57}$ They found that the stabilization of the $S_{1} \pi \pi^{*}$ bright state and the much faster decay to the ground state, when compared to the gas phase, should be related to the intermolecular hydrogen bonds with the solvent, which partially prevent the internal conversion to a dark $\mathrm{n} \pi^{*}$ state. For CMe and $\mathrm{SMe}$, both experimental and computational results show a weak solvent dependence in the absorption maximum wavelength. Yet, transient absorption spectra show a weak dependence with the solvent in the photodynamics of $\mathrm{CMe}$ and SMe in ethanol and CCT (see Table 3). Therefore, the use of implicit solvation seems to be suitable for the purposes of this work.

Table 3. Summary of the Theoretical ${ }^{a}$ and Experimental ${ }^{b}$ Time Constants for CMe and SMe

$\begin{array}{cccccc} & & \begin{array}{c}\text { theory } \\ (\text { mean })\end{array} & \begin{array}{c}\text { theory } \\ (\text { sigmoid })\end{array} & \begin{array}{c}\text { expt. } \\ \text { ethanol }\end{array} & \text { expt. CCT } \\ \mathrm{CMe} & \begin{array}{c}\tau_{\mathrm{CT}} \\ (\mathrm{fs})\end{array} & 334 \pm 14 & 572 & 190 \pm 40 & 220 \pm 40 \\ & \begin{array}{c}\tau_{\text {IC }} \\ (\mathrm{fs})\end{array} & 628 \pm 67 & 627 \pm 43 & 640 \pm 60 & 680 \pm 60 \\ \mathrm{SMe} & \begin{array}{c}\tau_{\mathrm{CT}} \\ (\mathrm{fs})\end{array} & 702 \pm 30 & 1279 & 350 \pm 40 & 300 \pm 40 \\ & \begin{array}{c}\tau_{\mathrm{IC}} \\ (\mathrm{fs})\end{array} & 1239 \pm 91 & 1422 \pm 115 & 1140 \pm 60 & 1450 \pm 60\end{array}$

${ }^{a}$ Present work. Theoretical values were obtained from excited-state dynamics with TD- $\omega$ B97XD/cc-pVDZ in ethanol (PCM). ${ }^{b}$ Experimental values from TEAS at concentrations of $1 \mathrm{mM}$ reported in reference. $^{22}$

Spectrum Simulations and Initial Conditions. We performed excited-state dynamics simulations to understand the time evolution of $\mathrm{CMe}$ and $\mathrm{SMe}$ and correlate the experimental and theoretical time constants. We began by computing the absorption spectra for CMe and SMe (Figure 5), from where the initial conditions for dynamics were selected.

The absorption spectra show that the first absorption band peaked at 3.85 and $3.53 \mathrm{eV}$ for CMe and SMe, respectively, in fair agreement with the experimental results $(3.30 \mathrm{eV}$ for $\mathrm{CMe}$ and $3.05 \mathrm{eV}$ for $\mathrm{SMe})$. These molecules present a large absorption cross section and absorb within the 315-400 nm region, as required for a UVA filter. ${ }^{58,59}$

The maximum absorption peak corresponds to the bright $\pi \pi^{*}$ $S_{1}$ state for both molecules. For CMe, the bright $\pi \pi^{*}$ is well separated from the $S_{2}$ and $S_{3}$ states, while for $S M e$, the $S_{2}$ state, also a $\pi \pi^{*}$ but with a small oscillator strength, appears as a shoulder around $4.0 \mathrm{eV}$. For this reason, for SMe we performed surface-hopping dynamics starting from the $S_{1}$ state but allowing hops to the $S_{2}$ and $S_{3}$ states, while for CMe we performed adiabatic dynamics on the $S_{1}$ surface. The spectral window selected for the dynamics was $3.5 \pm 0.3 \mathrm{eV}$ for $\mathrm{CMe}$ and $3.8 \pm$ 0.3 for $\mathrm{SMe}$, as indicated by the shaded area in Figure 5 .

Dynamics of Coumaryl Meldrum. We start the discussion with the analysis of $\mathrm{CMe}$ dynamics. Experimentally, two subpicosend time constants were extracted from the transient electronic absorption (TEA) spectrum using a sequential kinetic model. ${ }^{22}$ The extracted time constants were fitted as $\tau_{1}=190 \pm$ $40 \mathrm{fs}$ and $\tau_{2}=450 \pm 50 \mathrm{fs}$ in ethanol and $\tau_{1}=220 \pm 40 \mathrm{fs}$ and $\tau_{2}=$ $460 \pm 50 \mathrm{fs}$ in CCT. The internal conversion lifetime is approximately $\tau_{\mathrm{IC}}=\tau_{1}+\tau_{2}$ (Table 3 ).

Figure 6 shows the $S_{1}$ occupation, defined as the fraction of trajectories in the $S_{1}$ state as a function of the time during the adiabatic dynamics of CMe (see also Figure S11 for the occupation of the other states). One can see that there is a plateau in the first $\sim 300$ fs of the simulation before the deactivation to the ground state starts. Interestingly, we were not able to fit this decay function using either single- or multiexponential decay functions, as initially expected, but we found out that the $S_{1}$ occupation is well fitted by the sigmoid function

$$
p(t)=\frac{a}{1+\exp \left(\frac{t-\tau_{\mathrm{L}}}{\tau_{\mathrm{E}}}\right)}
$$

This function shows that the decay happens in two steps. First, an intermediate excited-state species must be formed within $\tau_{\mathrm{L}}$, and then the ground state is exponentially populated from this intermediate population within $\tau_{\mathrm{E}} \cdot a$ is the total variation of the population. The internal conversion lifetime, corresponding to the time to reduce the excited-state occupation to $1 / e$, is

$$
\tau_{\text {IC }} \equiv \tau_{E} \ln (e-1)+\tau_{L}
$$

The margin of error for the lifetime is

$$
\delta \tau=Z \frac{s}{\sqrt{N}}
$$

where $s$ is the distribution's standard deviation, $N$ is the number of trajectories, and $Z=1.96$ for the $95 \%$ confidence interval (assuming the validity of the central limit theorem). For the logistic distribution, $s=\left(\pi^{2} \tau_{\mathrm{E}}^{2} / 3\right)^{1 / 2}$. With these definitions, the
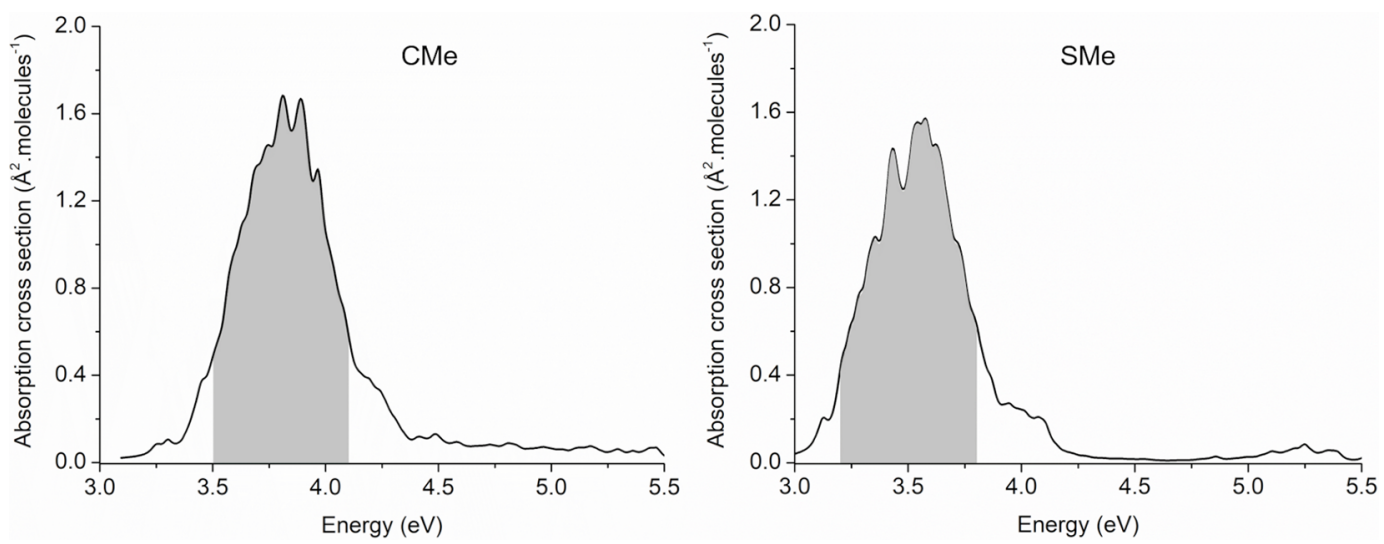

Figure 5. Simulated spectra of CMe (left) and SMe (right) calculated at TD- $\omega$ B97XD/cc-pVDZ in PCM/ethanol. Shaded area indicates the spectral window from where the initial conditions for the dynamics were selected. 

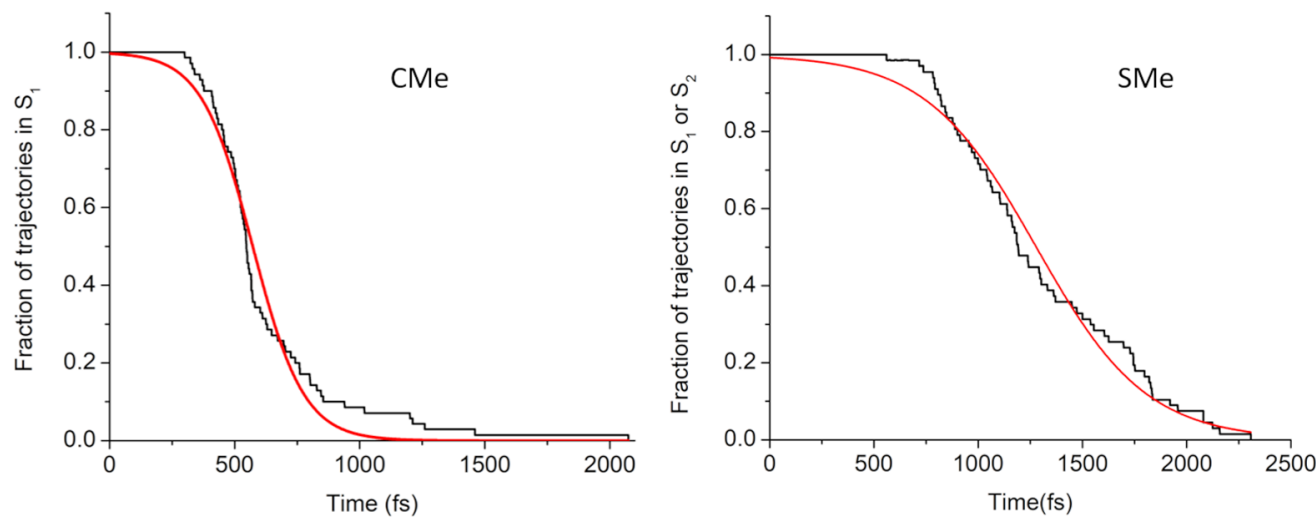

Figure 6. Fractional occupation of trajectories in the $S_{1}$ state as a function of time obtained for CMe (left) and SMe (right).

data fitting rendered $\tau_{\mathrm{L}}=572 \mathrm{fs}, \tau_{\mathrm{E}}=102 \mathrm{fs}$, and $\tau_{\mathrm{IC}}=627 \pm 43 \mathrm{fs}$ for CMe (Table 3).

Sigmoid fittings of the photophysical decay are uncommon. However, we found few other examples that would likely be well described by this functional form. ${ }^{60}$ Although controversial, ${ }^{61} \mathrm{a}$ sigmoid fitting has also been proposed as the basis of the nonphotochemical quenching (NPQ) of chlorophyll in plant leaves, ${ }^{62}$ the mechanism responsible for the dissipation of excess of photoenergy as heat. ${ }^{63}$

The sigmoid shape of the excited-state occupation (Figure 6) implies that the dynamics is governed by a logistic model (see Supporting Information Section 3), and as such, the exponential decay to the ground state depends on the accumulation of a critical population in an intermediate excited-state species. In our simulations, this intermediate species is the $S_{1 \text {-TICT }}$ minimum. Its barrierless formation is accompanied by a large geometrical change and decreased the $S_{1} / S_{0}$ energy gap. This relaxation was observed for all trajectories, and a representative one is illustrated in Figure S12. This figure shows that following the excitation into the ${ }^{1} \pi \pi^{*}$ state, the $S_{1} / S_{0}$ energy gap quickly drops to $\sim 0.5 \mathrm{eV}$ around $300 \mathrm{fs}$. This gap is consistent with that found for the $S_{1 \text {-TICT }}$ minimum at the calculation level used for the dynamics $(0.53 \mathrm{eV})$.

By monitoring a few key geometrical parameters in the trajectory ensemble, we could confirm that the small-gap region $(\sim 0.5 \mathrm{eV})$ is associated with the $S_{1 \text {-TICT }}$ minimum. In particular, the mean values of the $\mathrm{C} 1-\mathrm{C} 2-\mathrm{C} 3-\mathrm{C} 4$ torsional angle and the $\mathrm{C} 2-\mathrm{C} 3$ bond length are $93 \pm 12^{\circ}$ and $1.457 \pm 0.052 \AA$, which are comparable to the coordinates of the $S_{1-\text { TICT }}$ minimum (95.3 $3^{\circ}$ and $1.444 \AA$ ).

For a quantitative assignment of the time $\tau_{\mathrm{CT}}$ for $S_{1 \text {-TICT }}$ formation, we could take $\tau_{\mathrm{L}}$ from the sigmoid fitting, 572 fs. Nevertheless, this value is much longer than the experimental $\tau_{1}$ $(190 \pm 40 \mathrm{fs}$ in ethanol and $220 \pm 40 \mathrm{fs}$ in CCT, Table 3$)$. The problem with $\tau_{\mathrm{L}}$ is that because it is defined by the time when the excited-state population drops to one-half, it reflects not only the relaxation into the $S_{1-\text { TICT }}$ minimum but also part of the internal conversion to $S_{0}$. Thus, we opted to estimate the $\tau_{\mathrm{CT}}$ time for $S_{1-\text { TICT }}$ formation from the time needed for the trajectories to reach an $S_{1} / S_{0}$ gap smaller than $0.55 \mathrm{eV}$. In this way, we found that the mean time to reach this point is $\tau_{\mathrm{CT}}=334 \pm 14 \mathrm{fs}$, which correlates better with the first experimental time constant $\tau_{1}$.

Either way, $\tau_{1}$ is still significantly smaller than $\tau_{\mathrm{CT}}$, suggesting that the reason for the divergence may be in the somewhat different meaning of these quantities. The theoretical $\tau_{\mathrm{CT}}$ tells the time to populate the $S_{1 \text {-TICT }}$ minimum. In contrast, the experimental time constant $\tau_{1}$ measures more precisely when the excited-state absorption from the $S_{1}$ state into a highly excited state vanishes. The short $\tau_{1}$ value indicates that the reduction of the excited-state absorption happens before the TICT minimum is populated. Indirect evidence supporting this hypothesis is the small difference between $\tau_{1}$ in ethanol and CCT, $30 \mathrm{fs}$. As the viscosity of CCT is $\sim 20$ times larger than that of ethanol, if $\tau_{1}$ expresses the large conformational changes needed to populate TICT, it would be much longer in CCT.

After the $S_{1 \text {-TICT }}$ minimum is reached, we observe that all trajectories move in a configurational region where the energy gap with the ground state remains small (as illustrated in Figure S12). This small-gap region persists for $\sim 300 \mathrm{fs}$ until the trajectory converts to $S_{0}$. As mentioned, the sigmoid fitting tells that the internal conversion lifetime (relaxation to $S_{1 \text {-TICT }}$ minimum plus motion in the small-gap region) is $\tau_{\mathrm{IC}}=627 \pm$ $43 \mathrm{fs}$, in excellent agreement with the experimental value (640 \pm $60 \mathrm{fs}$ found in ethanol). ${ }^{22}$ Alternatively, we can also directly estimate the internal conversion lifetime by computing the mean time when the trajectories stopped due to a very small gap with the ground state (our proxy for $S_{1}-S_{0}$ conversion, as explained in the Computational Details). In this case, $\tau_{\mathrm{IC}}=628 \pm 67 \mathrm{fs}$, also showing excellent agreement with the experimental time constant.

During the motion in the small-gap region, we do not observe large geometrical changes but we do observe substantial oscillations in the bond lengths as well as ring puckering and pyramidalization of the $\mathrm{C} 3$ atom. Moreover, these nuclear motions are typical of photoisomerization reactions without chemical bond breaking or bond formation. ${ }^{64-66}$ This result is consistent with the topography found in our static calculations, which predicts a sloped $S_{1} / S_{0}$ intersection, with some small activation energy to be overcome.

Dynamics of Sinapoyl Meldrum. Similarly to CMe, the dynamics simulations of SMe revealed that the torsion around the allylic double bond (following $\mathrm{C} 2-\mathrm{C} 3$ stretching) is the main coordinate for $S_{1}$ relaxation from the vertically excited ${ }^{1} \pi \pi^{*}$ minimum.

The experimental time constants $\tau_{1}$ and $\tau_{2}$ in SMe are $350 \pm$ 40 and $790 \pm 40 \mathrm{fs}$ in ethanol and $300 \pm 40$ and $1150 \pm 40 \mathrm{fs}$ in CCT. As before, the internal conversion lifetime is about $\tau_{1}+\tau_{2}$ (Table 3). These values are longer than in $\mathrm{CMe}$, indicating that the methoxy groups in SMe play a role in delaying the dynamics. In addition, the presence of hydrogen bondings with the solvent, reported to accelerate internal conversion and to quench fluorescence in molecules presenting TICT,${ }^{67}$ seems to have a major role for SMe than CMe dynamics. 

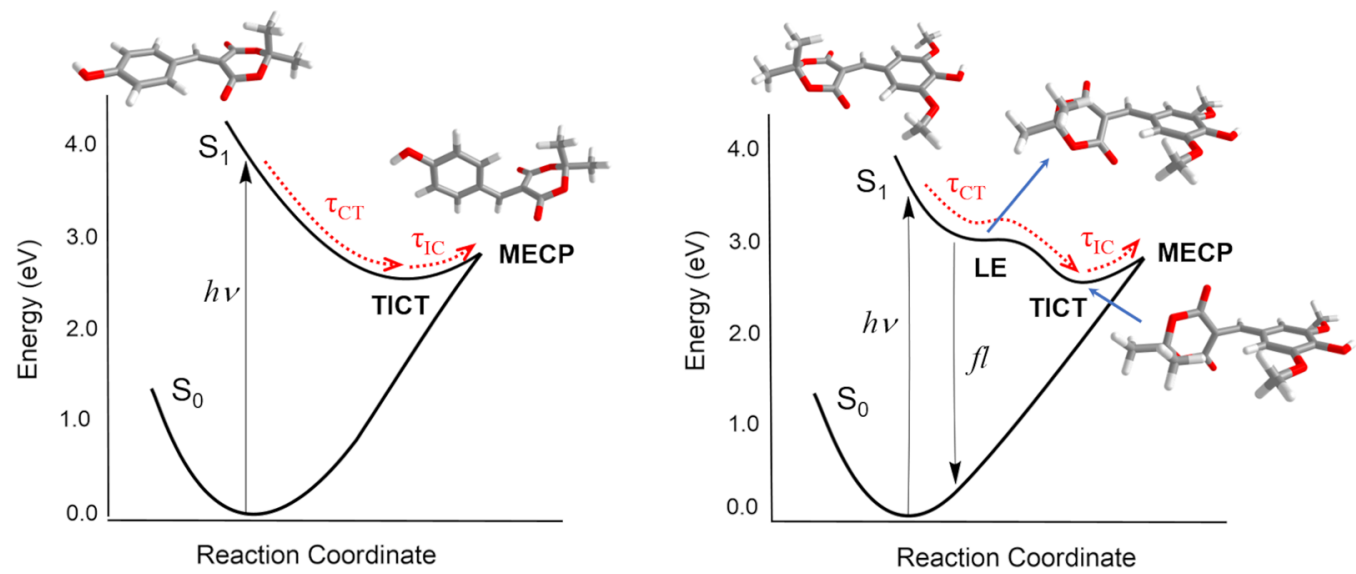

Figure 7. Schematic topography of the PES for CMe (left) and SMe (right).

Nonadiabatic excited-state dynamics also show that the photodeactivation of SMe takes longer than that of CMe. Monitoring the $S_{1}$ occupation as a function of time (Figure 6), one can see that the plateau at the beginning of the $\mathrm{SMe}$ simulations is longer and the $S_{1}$ depopulation is slower than in CMe. The average time for the molecules to reach the $S_{1 \text {-TICT }}$ minimum (measured as the mean value to reach an $S_{1} / S_{0}$ gap smaller than $0.6 \mathrm{eV}$ ) was $702 \pm 30 \mathrm{fs}$. During the motion in the small-gap region, the average $\mathrm{C} 1-\mathrm{C} 2-\mathrm{C} 3-\mathrm{C} 4$ torsional angle and $\mathrm{C} 2-\mathrm{C} 3$ bond length are $94 \pm 14^{\circ}$ and $1.457 \pm 0.05 \AA$ (Figure S13), similar to the values at the $S_{1-\text { TICT }}$ minimum (94.9 ${ }^{\circ}$ and $1.444 \AA$ ), confirming that the motion in this region corresponds to $S_{1-\text { TICT }}$ configurations. The time to reach the $\mathrm{S}_{1 \text {-TICT }}$ minimum in SMe, $\tau_{\mathrm{CT}}=702 \mathrm{fs}$, is about twice that, $334 \mathrm{fs}$, observed in $\mathrm{CMe}$. We attribute this delay to the presence of the shallow, partially twisted local $S_{1-\mathrm{LE}}$ minimum, which retards the dynamics (see also Figure S12).

SMe occupation is also well described by a sigmoid function (eq 1), delivering $\tau_{\mathrm{L}}=1279$ and $\tau_{\mathrm{E}}=264$, and therefore an internal conversion time of $\tau_{\text {IC }}=1422 \pm 115$ fs (Table 3). For $\mathrm{SMe}, 8 \%$ of the trajectories did not return to the ground state within the time window of 2 ps. This result is consistent with the photostability experiments, which revealed a loss of intensity in the normalized absorption spectrum of $2 \%$ for SMe (while for CMe it is only $0.9 \%){ }^{22}$ The average time for the other $92 \%$ of trajectories to return to the ground state allows for another estimate of the internal conversion time, $\tau_{\mathrm{IC}}=1239 \pm 91 \mathrm{fs}$. Both $\tau_{\mathrm{IC}}$ estimates match the experimental value of $1140 \mathrm{fs}$ obtained in the sequential model in ethanol as well.

Last, a stimulated emission at $\sim 475 \mathrm{~nm}$ and weak fluorescence signals were obtained for SMe with a time constant of $>2 \mathrm{~ns}$. As mentioned above, a small fraction of the population may be trapped in the $S_{1 \text {-TICT }}$ minimum and remain excited for longer times. However, this minimum is a dark state with an oscillator strength equal to zero, and it is very unlikely to fluoresce. Knowing that the main difference between the PECs for $\mathrm{CMe}$ and SMe is the additional LE minimum in the latter and that the LE to TICT energy barrier is only $<0.1 \mathrm{eV}$, would be this barrier sufficiently high to enable the weak molecular fluorescence?

To answer this question, we modeled the long-time-scale photophysics starting from the $S_{1-\mathrm{LE}}$ minimum (see Supporting Information, section 4). First, we determined that the fluorescence lifetime from the $S_{1-\mathrm{LE}}$ minimum $(448 \mathrm{~nm}$ ) should be about $\tau_{\mathrm{Fl}}=5.7 \mathrm{~ns}$, which qualitatively agrees with the experimental time constant $\left(\tau_{\mathrm{Fl}}>2 \mathrm{~ns}\right)$ and emission wavelength
$(475 \mathrm{~nm})$. Thus, assuming a small fluorescence quantum yield of $\varphi=1 \%$ (in line with the experiments), we estimated the time constant for SMe to cross the small energy barrier from $S_{1-\mathrm{LE}}$ to the $S_{1 \text {-TICT }}$ minimum as being about $\tau_{\mathrm{LE} \rightarrow \mathrm{CT}}=58 \mathrm{ps}$. This means that the SMe should be trapped in the $S_{1-\mathrm{LE}}$ minimum for about 58 ps to yield $1 \%$ of fluorescence. Given that the energy barrier should be about $0.1 \mathrm{eV}$, a 58 ps trapping could happen for reasonable values of reorganization energy and diabatic couplings of about $0.7 \mathrm{eV}$ and $50 \mathrm{~cm}^{-1}$, respectively. Therefore, given the qualitative agreement between the experimental and the calculated variables, we are confident that the weak fluorescence signal in $\mathrm{SMe}$ can be assigned to the $S_{1-\mathrm{LE}}$ minimum.

\section{CONCLUSIONS}

This work has presented an in-depth theoretical analysis of the excited-state photodynamics of $\mathrm{CMe}$ and $\mathrm{SMe}$, whose experimental results were discussed in a previous letter. ${ }^{22}$ Our theoretical calculations encompass the characterization of the potential energy surfaces at the TD-DFT and CASPT2 levels and excited-state dynamics simulations.

The photodynamics of both molecules is schematically illustrated in Figure 7. In summary, once excited into the bright $\mathrm{S}_{1}$ state, $\mathrm{CMe}$ and $\mathrm{SMe}$ evolve in the subpicoseconds scale to an $\sim 90^{\circ}$-twisted minimum near an intersection with the ground state. This minimum has a twisted intramolecular chargetransfer (TICT) character, coupling $S_{1}$ and $S_{0}$. From there, minor geometric changes tune an $S_{1} / S_{0}$ intersection around 0.2 $\mathrm{eV}$ higher. The sloped intersection seam enables a nonadiabatic transition, regenerating the ground state.

A Sigmoid function can nicely fit the excited-state populations. This means that the dynamics of CMe and SMe are driven by underlying logistic-decay kinetics, characterized by the population of an excited-state intermediate species, followed by an internal conversion from this intermediate. This intermediate species has been identified as the TICT $S_{1}$ minimum, populated within about $0.3 \mathrm{ps}$ in $\mathrm{CMe}$ and $0.7 \mathrm{fs}$ in $\mathrm{SMe}$. After this minimum is populated, both molecules still need a few hundreds of femtoseconds before the internal conversion to $S_{0}$ takes place. CMe returns to the ground state at about $0.6 \mathrm{ps}$ after the excitation, while SMe does it at about 1.2 ps. The internal conversion lifetimes are in excellent agreement with the experiment, especially for $\mathrm{CMe}$. An additional local $\mathrm{S}_{1}$ minimum for $\mathrm{SMe}$, with LE character, is responsible for the weak fluorescence experimentally observed in this molecule. 


\section{ASSOCIATED CONTENT}

\section{(s) Supporting Information}

The Supporting Information is available free of charge at https://pubs.acs.org/doi/10.1021/acs.jpca.1c03315.

Geometries, natural transition orbitals, CT numbers, reaction pathways, state occupations, analysis of exemplary trajectories, characterization of the gap closure, population kinetics, analysis of the trapping at the LE minimum, and Cartesian coordinates for the optimized geometries (PDF)

\section{AUTHOR INFORMATION}

\section{Corresponding Authors}

Josene M. Toldo - Aix Marseille Université, CNRS, ICR, Marseille 13397, France; ○ orcid.org/0000-0002-89696635; Email: josene-maria.toldo@univ-amu.fr

Mario Barbatti - Aix Marseille Université, CNRS, ICR, Marseille 13397, France; 이이.org/0000-0001-93366607; Email: mario.barbatti@univ-amu.fr

\section{Author}

Mariana T. do Casal - Aix Marseille Université, CNRS, ICR, Marseille 13397, France

Complete contact information is available at: https://pubs.acs.org/10.1021/acs.jpca.1c03315

\section{Notes}

The authors declare no competing financial interest.

\section{ACKNOWLEDGMENTS}

The authors are thankful for the support of the FetOpen grant BoostCrop (Grant agreement 828753) and the project Equip@ Meso (ANR-10-EQPX-29-01) funded by the French Government "Investissements d'Avenir" program for the computational resources. The authors thank Prof. V. Stravos, T. T. Abiola, and Dr. J. Woolley for discussions and Dr. M. Pinheiro Jr.

\section{REFERENCES}

(1) Armstrong, B. K.; Cust, A. E. Sun Exposure and Skin Cancer, and the Puzzle of Cutaneous Melanoma: A Perspective on Fears Et Al. Mathematical Models of Age and Ultraviolet Effects on the Incidence of Skin Cancer among Whites in the United States. Cancer Epidemiol. 2017, 48, 147-156.

(2) de Assis, L. V. M.; Tonolli, P. N.; Moraes, M. N.; Baptista, M. S.; de Lauro Castrucci, A. M. How Does the Skin Sense Sun Light? An Integrative View of Light Sensing Molecules. J. Photochem. Photobiol., C 2021, 47, 100403.

(3) Rodrigues, N. D.; Staniforth, M.; Stavros, V. G. Photophysics of Sunscreen Molecules in the Gas Phase: A Stepwise Approach Towards Understanding and Developing Next-Generation Sunscreens. Proc. $R$. Soc. London, Ser. A 2016, 472, 20160677.

(4) Frederick, J. E.; Snell, H. E.; Haywood, E. K. Solar Ultraviolet Radiation at the Earth's Surface. Photochem. Photobiol. 1989, 50, 443450.

(5) Sinha, R. P.; Häder, D.-P. Uv-Induced DNA Damage and Repair: A Review. Photochem. Photobiol. Sci. 2002, 1, 225-236.

(6) Encinas Perea, S. Solar Filters: A Strategy of Photoprotection. In Applied Photochemistry: When Light Meets Molecules; Bergamini, G., Silvi, S., Eds.; Springer: Cham, 2016; Vol. 92, pp 459-478.

(7) Battie, C.; Jitsukawa, S.; Bernerd, F.; Del Bino, S.; Marionnet, C.; Verschoore, M. New Insights in Photoaging, Uva Induced Damage and Skin Types. Exp. Dermatol. 2014, 23, 7-12.

(8) Afonso, S.; Horita, K.; Sousa e Silva, J.P.; Almeida, I.F.; Amaral, M.H.; Lobao, P.A.; Costa, P.C.; Miranda, M. S.; Esteves da Silva, J.
C.G.; Sousa Lobo, J.M. Photodegradation of Avobenzone: Stabilization Effect of Antioxidants. J. Photochem. Photobiol., B 2014, 140, 36-40.

(9) Mturi, G. J.; Martincigh, B. S. Photostability of the Sunscreening Agent 4-Tert-Butyl-4'-Methoxydibenzoylmethane (Avobenzone) in Solvents of Different Polarity and Proticity. J. Photochem. Photobiol., A 2008, 200, 410-420.

(10) Jansen, M. A. K.; Gaba, V.; Greenberg, B. M. Higher Plants and UV-B Radiation: Balancing Damage, Repair and Acclimation. Trends Plant Sci. 1998, 3, 131-135.

(11) Dean, J. C.; Kusaka, R.; Walsh, P. S.; Allais, F.; Zwier, T. S. Plant Sunscreens in the UV-B: Ultraviolet Spectroscopy of Jet-Cooled Sinapoyl Malate, Sinapic Acid, and Sinapate Ester Derivatives. J. Am. Chem. Soc. 2014, 136, 14780-14795.

(12) Baker, L. A.; Horbury, M. D.; Greenough, S. E.; Allais, F.; Walsh, P. S.; Habershon, S.; Stavros, V. G. Ultrafast Photoprotecting Sunscreens in Natural Plants. J. Phys. Chem. Lett. 2016, 7, 56-61.

(13) Chapple, C. C.; Vogt, T.; Ellis, B. E.; Somerville, C. R. An Arabidopsis Mutant Defective in the General Phenylpropanoid Pathway. Plant Cell 1992, 4, 1413-1424.

(14) Baker, L. A.; Marchetti, B.; Karsili, T. N.; Stavros, V. G.; Ashfold, M. N. Photoprotection: Extending Lessons Learned from Studying Natural Sunscreens to the Design of Artificial Sunscreen Constituents. Chem. Soc. Rev. 2017, 46, 3770-3791.

(15) Horbury, M.; Flourat, A.; Greenough, S.; Allais, F.; Stavros, V. Investigating Isomer Specific Photoprotection in a Model Plant Sunscreen. Chem. Commun. 2018, 54, 936-939.

(16) Abiola, T. T.; Whittock, A. L.; Stavros, V. G. Unravelling the Photoprotective Mechanisms of Nature-Inspired Ultraviolet Filters Using Ultrafast Spectroscopy. Molecules 2020, 25 (17), 3945.

(17) Zhao, X.; Ji, F.; Liang, Y.; Li, P.; Jia, Y.; Feng, X.; Sun, Y.; Shi, Y.; Zhu, L.; Zhao, G. Theoretical and Spectroscopic Investigation on Ultrafast Nonadiabatic Photoprotective Mechanism of Novel Ultraviolet Protective Compounds Inspired by Natural Sunscreens. J. Lumin. 2020, 223, 117228.

(18) Baker, L. A.; Staniforth, M.; Flourat, A. L.; Allais, F.; Stavros, V. G. Gas-Solution Phase Transient Absorption Study of the Plant Sunscreen Derivative Methyl Sinapate. ChemPhotoChem. 2018, 2, $743-748$.

(19) Kockler, J.; Oelgemöller, M.; Robertson, S.; Glass, B. D. Photostability of Sunscreens. J. Photochem. Photobiol., C 2012, 13, 91110.

(20) Sharma, A.; Bányiová, K.; Babica, P.; El Yamani, N.; Collins, A. R.; Cupr, P. Different DNA Damage Response of Cis and Trans Isomers of Commonly Used Uv Filter after the Exposure on Adult Human Liver Stem Cells and Human Lymphoblastoid Cells. Sci. Total Environ. 2017, 593, 18-26.

(21) Horbury, M. D.; Holt, E. L.; Mouterde, L. M.; Balaguer, P.; Cebrián, J.; Blasco, L.; Allais, F.; Stavros, V. G. Towards Symmetry Driven and Nature Inspired Uv Filter Design. Nat. Commun. 2019, 10, 4748.

(22) Abiola, T. T.; Rodrigues, N. d. N.; Ho, C.; Coxon, D. J. L.; Horbury, M. D.; Toldo, J. M.; do Casal, M. T.; Rioux, B.; Peyrot, C.; Mention, M. M.; et al. New Generation UV-A Filters: Understanding Their Photodynamics on a Human Skin Mimic. J. Phys. Chem. Lett. 2021, 12, 337-344.

(23) Chai, J.-D.; Head-Gordon, M. Long-Range Corrected Hybrid Density Functionals with Damped Atom-Atom Dispersion Corrections. Phys. Chem. Chem. Phys. 2008, 10, 6615-6620.

(24) Becke, A. D. Density-Functional Exchange-Energy Approximation with Correct Asymptotic Behavior. Phys. Rev. A: At., Mol., Opt. Phys. 1988, 38, 3098-3100.

(25) Lee, C.; Yang, W.; Parr, R. G. Development of the Colle-Salvetti Correlation-Energy Formula into a Functional of the Electron Density. Phys. Rev. B: Condens. Matter Mater. Phys. 1988, 37, 785-789.

(26) Grimme, S.; Antony, J.; Ehrlich, S.; Krieg, H. A Consistent and Accurate $\mathrm{Ab}$ Initio Parametrization of Density Functional Dispersion Correction (DFT-D) for the 94 Elements H-Pu. J. Chem. Phys. 2010, $132,154104$. 
(27) Dunning, T. H. Gaussian Basis Sets for Use in Correlated Molecular Calculations. I. The Atoms Boron through Neon and Hydrogen. J. Chem. Phys. 1989, 90, 1007-1023.

(28) Mennucci, B.; Cancès, E.; Tomasi, J. J. Phys. Chem. B 1997, 101, 10506 .

(29) Frisch, M. J.; Trucks, G. W.; Schlegel, H. B.; Scuseria, G. E.; Robb, M. A.; Cheeseman, J. R.; Scalmani, G.; Barone, V.; Petersson, G. A.; Nakatsuji, H., et al. Gaussian 16, Revision C.01; Gaussian, Inc.: Wallingford, CT, 2016.

(30) Levine, B. G.; Coe, J. D.; Martínez, T. J. Optimizing Conical Intersections without Derivative Coupling Vectors: Application to Multistate Multireference Second-Order Perturbation Theory (MsCaspt2). J. Phys. Chem. B 2008, 112, 405-413.

(31) Levine, B. G.; Ko, C.; Quenneville, J.; MartÍnez, T. J. Conical Intersections and Double Excitations in Time-Dependent Density Functional Theory. Mol. Phys. 2006, 104, 1039-1051.

(32) Fogarasi, G.; Zhou, X.; Taylor, P. W.; Pulay, P. The Calculation of Ab Initio Molecular Geometries: Efficient Optimization by Natural Internal Coordinates and Empirical Correction by Offset Forces. J. Am. Chem. Soc. 1992, 114, 8191-8201.

(33) Malmqvist, P.-Å.; Roos, B. O. The CASSCF State Interaction Method. Chem. Phys. Lett. 1989, 155, 189-194.

(34) Finley, J.; Malmqvist, P.-Å.; Roos, B. O.; Serrano-Andrés, L. The Multi-State CASPT2Method. Chem. Phys. Lett. 1998, 288, 299-306.

(35) Widmark, P.-O.; Malmqvist, P.-Å.; Roos, B. O. Density Matrix Averaged Atomic Natural Orbital (ANO) Basis Sets for Correlated Molecular Wave Functions. Theor. Chim. Acta 1990, 77, 291-306.

(36) Ghigo, G.; Roos, B. O.; Malmqvist, P.-Å. A Modified Definition of the Zeroth-Order Hamiltonian in Multiconfigurational Perturbation Theory (CASPT2). Chem. Phys. Lett. 2004, 396, 142-149.

(37) Forsberg, N.; Malmqvist, P.-Å. Multiconfiguration Perturbation Theory with Imaginary Level Shift. Chem. Phys. Lett. 1997, 274, 196204.

(38) Fdez Galván, I.; Vacher, M.; Alavi, A.; Angeli, C.; Aquilante, F.; Autschbach, J.; Bao, J. J.; Bokarev, S. I.; Bogdanov, N. A.; Carlson, R. K.; et al. Openmolcas: From Source Code to Insight. J. Chem. Theory Comput. 2019, 15, 5925-5964.

(39) Plasser, F.; Lischka, H. Analysis of Excitonic and Charge Transfer Interactions from Quantum Chemical Calculations. J. Chem. Theory Comput. 2012, 8, 2777-2789.

(40) Crespo-Otero, R.; Barbatti, M. Spectrum Simulation and Decomposition with Nuclear Ensemble: Formal Derivation and Application to Benzene, Furan and 2-Phenylfuran. Theor. Chem. Acc. 2012, 131, 1237.

(41) Barbatti, M.; Granucci, G.; Persico, M.; Ruckenbauer, M.; Vazdar, M.; Eckert-Maksic, M.; Lischka, H. The on-the-Fly SurfaceHopping Program System Newton-X: Application to Ab Initio Simulation of the Nonadiabatic Photodynamics of Benchmark Systems. J. Photochem. Photobiol., A 2007, 190, 228-240.

(42) Frisch, M. J.; Trucks, G. W.; Schlegel, H. B.; Scuseria, G. E.; Robb, M. A.; Cheeseman, J. R.; Scalmani, G.; Barone, V.; Petersson, G. A.; Nakatsuji, H., et al. Gaussian 09, Revision D.01; Gaussian, Inc.: Wallingford, CT, 2009.

(43) Swope, W. C.; Andersen, H. C.; Berens, P. H.; Wilson, K. R. A Computer Simulation Method for the Calculation of Equilibrium Constants for the Formation of Physical Clusters of Molecules: Application to Small Water Clusters. J. Chem. Phys. 1982, 76, 637-649.

(44) Granucci, G.; Persico, M. Critical Appraisal of the Fewest Switches Algorithm for Surface Hopping. J. Chem. Phys. 2007, 126, 134114.

(45) Tully, J. C. Molecular Dynamics with Electronic Transitions. J. Chem. Phys. 1990, 93, 1061-1071.

(46) Dreuw, A.; Weisman, J. L.; Head-Gordon, M. Long-Range Charge-Transfer Excited States in Time-Dependent Density Functional Theory Require Non-Local Exchange. J. Chem. Phys. 2003, 119, 29432946.

(47) Fan, J.; Roeterdink, W.; Buma, W. J. Excited-State Dynamics of Isolated and (Micro)Solvated Methyl Sinapate: The Bright and Shady Sides of a Natural Sunscreen. Mol. Phys. 2021, 119, No. e1825850.
(48) Yamazaki, K.; Miyazaki, Y.; Harabuchi, Y.; Taketsugu, T.; Maeda, S.; Inokuchi, Y.; Kinoshita, S.-n.; Sumida, M.; Onitsuka, Y.; Kohguchi, H.; et al. Multistep Intersystem Crossing Pathways in Cinnamate-Based Uv-B Sunscreens. J. Phys. Chem. Lett. 2016, 7, 4001-4007.

(49) Muramatsu, S.; Nakayama, S.; Kinoshita, S.-n.; Onitsuka, Y.; Kohguchi, H.; Inokuchi, Y.; Zhu, C.; Ebata, T. Electronic State and Photophysics of 2-Ethylhexyl-4-Methoxycinnamate as Uv-B Sunscreen under Jet-Cooled Condition. J. Phys. Chem. A 2020, 124, 1272-1278.

(50) Tan, E. M. M.; Hilbers, M.; Buma, W. J. Excited-State Dynamics of Isolated and Microsolvated Cinnamate-Based UV-B Sunscreens. J. Phys. Chem. Lett. 2014, 5, 2464-2468.

(51) Barbatti, M.; Ruckenbauer, M.; Lischka, H. The Photodynamics of Ethylene: A Surface-Hopping Study on Structural Aspects. J. Chem. Phys. 2005, 122, 174307.

(52) Sellner, B.; Barbatti, M.; Müller, T.; Domcke, W.; Lischka, H. Ultrafast Non-Adiabatic Dynamics of Ethylene Including Rydberg States. Mol. Phys. 2013, 111, 2439-2450.

(53) Michl, J.; Bonacic-Koutecky, V. Electronic Aspects of Organic Photochemistry; Wiley-Interscience, 1990.

(54) Coyle, J. D.; Carless, H. A. J. Selected Aspects of Photochemistry. I Photochemistry of Carbonyl Compounds. Chem. Soc. Rev. 1972, 1, 465-480.

(55) El-Sayed, M. A. Triplet State. Its Radiative and Nonradiative Properties. Acc. Chem. Res. 1968, 1, 8-16.

(56) Englman, R.; Jortner, J. The Energy Gap Law for Radiationless Transitions in Large Molecules. Mol. Phys. 1970, 18, 145-164.

(57) Liu, F.; Du, L.; Lan, Z.; Gao, J. Hydrogen Bond Dynamics Governs the Effective Photoprotection Mechanism of Plant Phenolic Sunscreens. Photochem. Photobiol. Sci. 2017, 16, 211-219.

(58) Rodrigues, N. D. N.; Staniforth, M.; Young, J. D.; Peperstraete, Y.; Cole-Filipiak, N. C.; Gord, J. R.; Walsh, P. S.; Hewett, D. M.; Zwier, T. S.; Stavros, V. G. Towards Elucidating the Photochemistry of the Sunscreen Filter Ethyl Ferulate Using Time-Resolved Gas-Phase Spectroscopy. Faraday Discuss. 2016, 194, 709-729.

(59) Shaath, N. A. Ultraviolet Filters. Photochem. Photobiol. Sci. 2010, 9, 464-469.

(60) Malhado, J. P.; Spezia, R.; Hynes, J. T. Conical Intersection Structure and Dynamics for a Model Protonated Schiff Base Photoisomerization in Solution. Int. J. Quantum Chem. 2013, 113, 296-305.

(61) Holzwarth, A. R.; Lenk, D.; Jahns, P. On the Analysis of NonPhotochemical Chlorophyll Fluorescence Quenching Curves: I. Theoretical Considerations. Biochim. Biophys. Acta, Bioenerg. 2013, 1827, 786-792.

(62) Ruban, A. V.; Wentworth, M.; Horton, P. Kinetic Analysis of Nonphotochemical Quenching of Chlorophyll Fluorescence. 1. Isolated Chloroplasts. Biochemistry 2001, 40, 9896-9901.

(63) Duffy, C. D. P.; Ruban, A. V. Dissipative Pathways in the Photosystem-II Antenna in Plants. J. Photochem. Photobiol., B 2015, $152,215-226$.

(64) Kumpulainen, T.; Lang, B.; Rosspeintner, A.; Vauthey, E. Ultrafast Elementary Photochemical Processes of Organic Molecules in Liquid Solution. Chem. Rev. 2017, 117, 10826-10939.

(65) Taylor, M. A.; Zhu, L.; Rozanov, N. D.; Stout, K. T.; Chen, C.; Fang, C. Delayed Vibrational Modulation of the Solvated GFP Chromophore into a Conical Intersection. Phys. Chem. Chem. Phys. 2019, 21, 9728-9739.

(66) Weigel, A.; Ernsting, N. P. Excited Stilbene: Intramolecular Vibrational Redistribution and Solvation Studied by Femtosecond Stimulated Raman Spectroscopy. J. Phys. Chem. B 2010, 114, 78797893.

(67) Zhao, G.-J.; Han, K.-L. Hydrogen Bonding in the Electronic Excited State. Acc. Chem. Res. 2012, 45, 404-413. 